\title{
Von den Nachbarn lernen? Care-Regime in Deutschland, Österreich und Frankreich
}

Sigrid Leitner

Kinderbetreuungs- und Altenpflegepolitiken führen in den Sozialversicherungsländern - Deutschland, Österreich und Frankreich zu geschlechts- und schichtspezifischen Diskriminierungsstrukturen gemessen an den Zielen einer eigenständigen Existenzsicherung in der Betreuungs- und Pflegezeit, der partnerschaftlichen Aufteilung der Care-Aufgaben und den Wiedereinstiegschancen in den Arbeitsmarkt. Jüngere Reformen in diesen Ländern zeigen jedoch einen Trend zur Modernisierung. Welche Maßnahmen können bestehende Ungleichheiten verringern? Welche Lehren kann Deutschland aus den Erfahrungen seiner Nachbarn ziehen? ${ }^{1}$

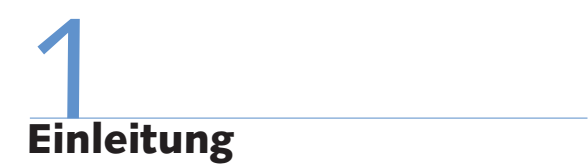

Alle modernen Wohlfahrtsstaaten sehen sich heute den Problemen steigender Betreuungs- und Pflegebedarfe gegenüber. Während auf der einen Seite veränderte Familienstrukturen und die Zunahme der Müttererwerbstätigkeit nach mehr Kinderbetreuungsplätzen verlangen, führt auf der anderen Seite die Alterung der Bevölkerung zu einem erhöhten Pflegeaufwand. Im Zuge des Ausbaus der europäischen Wohlfahrtsstaaten nach dem Zweiten Weltkrieg wurde - in manchen Ländern stärker, in anderen schwächer - ein System von Familienarbeit institutionalisiert, das Frauen ermöglichte (und gleichzeitig von ihnen einforderte), für ihre Kinder, ältere Familienmitglieder und Behinderte zu Hause zu sorgen (Knijn/Kremer 1997). Diese strukturelle Verankerung des männlichen Ernährermodells wirkt bis heute nach und wird seit Ende der 1970er/Anfang der 1980er Jahre zunehmend durch direkte Geldleistungen für Familienarbeit ergänzt. So haben im Bereich der Kinderbetreuung die meisten EU-Länder ErziehungszeitRegelungen eingeführt oder ausgeweitet, die oftmals mit einer allerdings geringen Geldleistung verbunden sind. In vielen Ländern werden Erziehungs- und Pflegezeiten im Rentensystem anerkannt und familiale Pflegearrangements durch Geldleistungen unterstützt.

Abgesehen von dieser generellen Entwicklung ist die Bedeutung der Familie in der Erbringung von Betreuungs- und Pflegearbeiten in den einzelnen Wohlfahrtsstaaten unterschiedlich stark: Die Familie „konkurriert“ gewissermaßen mit anderen, öffentlich subventionierten oder markt- förmig organisierten Erbringern sozialer Dienstleistungen. Die jeweils spezifische Verortung der Betreuungs- und Pflegearbeit zwischen den vier „Orten der Wohlfahrtsproduktion" - Staat, dritter Sektor, Markt und Familie - charakterisiert das Care-Regime eines Wohlfahrtsstaats. Die Bismarckschen Sozialversicherungsstaaten kontinentaleuropäischer Prägung zeichnen sich dadurch aus, dass sie der Familie einen besonders großen Stellenwert bei der Erbringung von Betreuungs- und Pflegearbeiten zumessen: Auf der Grundlage einer nur schwach ausgeprägten Dienstleistungsinfrastruktur wird entweder davon ausgegangen, dass die Familie diese Arbeitsleistung von sich aus erbringt, oder der Rückgriff auf familiale Pflege und Betreuung wird durch sozialpolitische Maßnahmen sogar aktiv unterstützt.

Diesem besonderen Fokus auf die Familie als zentrale Institution für Kinderbetreuung und Altenpflege liegt ein spezifisches Familien- und Geschlechterleitbild zugrunde, nämlich das Ernährer-Hausfrau-Modell. Sozialpolitische Maßnahmen, die darauf zielen, Pflege- und Betreuungsarbeit in der Familie zu verankern, nehmen automatisch Einfluss auf das Geschlechterverhältnis. Da familiale Pflege- und Betreuungsarbeit in den meisten Fällen von Frauen erbracht wird, unterstützen derartige Maßnahmen nicht nur die Familie als solche, sondern auch die damit verbundene Festlegung von Frauen auf die Familienarbeit (Jenson/Jacobzone 2000). Ungerson (1995) gibt zu bedenken, dass bereits kleine, symbolische Leistungen Frauen unter Druck setzen, aus der Erwerbsarbeit auszusteigen, um Familienarbeit zu leisten. Dieser Zusammenhang stellt keinen Automatismus, sondern eher eine Dilemma-Situation dar, aus der es durchaus Auswege geben kann: Es können Anreize für eine geschlechtergerechte Aufteilung von Familienarbeit gesetzt und Übergänge zwischen Familien- und Erwerbsarbeit unterstützt werden. Angesichts der gesellschaftlichen Umbrüche hinsichtlich der gelebten Geschlechternormen stellt sich die Frage, inwiefern die bestehenden Kinderbetreuungs- und Altenpflegepolitiken zeitgemäß sind. Die jüngere deutsche Familienpolitik hat hier sicherlich Reformschritte in die richtige Richtung gesetzt. Der Vergleich mit Österreich und Frankreich soll verdeutlichen, wo weitere Reformpotenziale liegen könnten und welche Wege weniger empfehlenswert sind. Maßstab hierfür ist gemäß der feministischen vergleichenden Wohlfahrtsstaatsforschung (Bruning/Platenga 1999) die Frage, inwiefern es erstens gelingt, eine eigenständige Existenzsicherung für die Pflegenden und Betreuenden zu gewähren und zweitens deren Wiedereinstiegschancen in den Arbeitsmarkt zu fördern. Drittens sind aus der Sicht der Geschlechtergerechtigkeit die Anreize für eine partnerschaftliche Arbeitsteilung zentral.

Neben der Analyse der geschlechtsspezifischen Struktureffekte unterschiedlicher Care-Regime soll im Folgenden auch nach den schichtspezifischen Wirkungen von

\footnotetext{
Der Aufsatz referiert zentrale Ergebnisse meiner Habilitationsschrift (Leitner 2008), die im September 2008 an der Sozialwissenschaftlichen Fakultät der Universität Göttingen eingereicht wurde.

Sigrid Leitner, Prof. Dr., Professorin für Sozialpolitik an der Fachhochschule Köln. Arbeitsschwerpunkte: International vergleichende Wohlfahrtsstaatsforschung, Sozialpolitik als Geschlechterpolitik, Renten- und Familienpolitik. e-Mail: sigrid.leitner@fh-koeln.de
} 
Kinderbetreuungs- und Altenpflegepolitiken für die Familienarbeitenden gefragt werden. Letzteren werden durch sozialpolitische Maßnahmen der Kinderbetreuung und Altenpflege Handlungsspielräume eröffnet: Zum einen wird z. B. durch Transferleistungen Familienarbeit in vielen Fällen überhaupt erst ermöglicht, zum anderen entstehen z. B. durch den Ausbau von sozialen Diensten Möglichkeiten zur Auslagerung von Familienarbeit an andere Leistungserbringer. Wer in welchem Maße von diesen Optionen profitieren kann, hängt allerdings von der konkreten Ausgestaltung der Maßnahmen ab.

Im Folgenden werden zunächst die Kinderbetreuungspolitiken (Abschnitt 2) und in einem zweiten Schritt die Altenpflegepolitiken (Abschnitt 3) der drei Vergleichsländer dargestellt und auf ihre geschlechtsund schichtspezifischen Wirkungen hin analysiert. Das Schlusskapitel (Abschnitt 4) verdichtet die Analyseergebnisse zu politischen Handlungsempfehlungen.

\section{Kinderbetreuungspolitiken in den Sozialversicherungs- ländern}

In der vergleichenden Familienpolitikforschung gilt Frankreich als vorbildlich hinsichtlich der Möglichkeiten, Familie und Beruf zu vereinbaren. Bereits für UnterDreijährige ist die Betreuungsinfrastruktur sehr gut ausgebaut. In Deutschland und Österreich hingegen fehlen vergleichbare Angebote, allerdings wird in Deutschland der Ausbau der Kleinkinderbetreuung gerade forciert. In allen drei Ländern existieren zudem bezahlte Elternzeitregelungen, die zu unterschiedlichen geschlechts- und schichtspezifischen Effekten führen, indem sie tradierte Geschlechterrollen fördern oder nur teilweise aufweichen und bestehende soziale Ungleichheiten eher verstärken.

\subsection{DEUTSCHLAND}

Seit 2007 ersetzt das neue Elterngeld das alte Erziehungsgeld. Nunmehr können Eltern maximal 14 Monate lang eine Lohnersatzleistung von $67 \%$ erhalten (Untergrenze: $300 €$, Obergrenze: $1.800 €)$. Zwei Monate des Elterngeldbezugs sind für den jeweils anderen Elternteil reserviert und verfallen, wenn dieser sie nicht in Anspruch nimmt. Für rund ein Viertel der Erziehenden besteht aufgrund der Orientierung der Leistungshöhe am vorangegangenen Einkommen während des Elterngeldbezugs keine finanzielle Abhängigkeit von einem Ernährer: 11,4 \% der Elterngeldbezieher und -bezieherinnen erhielten 2007 ein Elterngeld in Höhe von $750 €$ bis $1.000 €$ monatlich, bei $16,3 \%$ lag das Elterngeld über $1.000 €$ pro Monat. Das bedeutet im Umkehrschluss aber auch, dass das Elterngeld in $72,3 \%$ der Fälle weniger als $750 €$ ausmachte, bei $32 \%$ wurde sogar nur der Mindestbetrag von $300 €$ ausbezahlt (BMFSFJ 2008). Für Eltern mit vormals geringem Einkommen bietet das Elterngeld somit keine eigenständige Existenzsicherung. ${ }^{2}$ Die relativ kurze Bezugsdauer setzt einen Anreiz für kurze Erwerbsunterbrechungen mit einem raschen Wiedereinstieg in den Arbeitsmarkt, während die Ausgestaltung als Lohnersatzleistung gemeinsam mit den Partnermonaten die Beteiligung von Vätern an der Kinderbetreuung attraktiver macht. Insgesamt betrug die Väterbeteiligung 2007 10,5 \%, wobei $61 \%$ dieser Väter ausschließlich die beiden Partnermonate in Anspruch nahmen. Mütter beanspruchten hingegen das Elterngeld zu $87 \%$ für zwölf Monate (ebd.). Eine Veränderung der familialen Arbeitsteilung gegenüber der 5 \%igen Väterbeteiligung unter der alten Erziehungsgeldregelung wird somit nur bedingt erreicht (Ehnis 2008; Rüling 2008).

Nach der bezahlten Elternzeit kann wie bisher unbezahlte Elternzeit bis zum dritten Lebensjahr des Kindes beansprucht werden. Die Anreize zum schnellen Wiedereinstieg in den Arbeitsmarkt, die das neue Elterngeld setzt, werden somit teilweise durch die alte Elternzeitregelung konterkariert, sofern Eltern sich den langen Ausstieg leisten können. Wer tatsächlich rasch wieder erwerbstätig sein möchte oder muss, sieht sich in Westdeutschland derzeit noch einer relativ schwach entwickelten Betreuungsstruktur gegenüber. Im März 2008 hatten bundesweit $18 \%$ (im Westen $12 \%$, im Osten $42 \%$ ) der Unter-Dreijährigen einen Betreuungsplatz (Statistisches Bundesamt 2008). Der im Rahmen des Kinderförderungsgesetzes im April 2008 verabschiedete Ausbau der Kinderbetreuung sieht eine Betreuungsquote von $35 \%$ bis zum Jahr 2013 vor. Danach wird auch der Rechtsanspruch des Kindes auf einen Betreuungsplatz ab dem ersten Lebensjahr in Kraft treten.

\section{2 ÖSTERREICH}

In Österreich wurde 2002 die Geldleistung während der Elternzeit reformiert. Anspruch auf das neue Kinderbetreuungsgeld (KBG) haben erstmals alle Eltern unabhängig von vorangegangener Erwerbsarbeit. Die Höhe des KBG beträgt pauschal $436 €$ pro Monat, wobei gering verdienende Eltern einen Zuschuss zum KBG von $181,68 €$ pro Monat erhalten können. Die KBG-Bezugsdauer ist gegenüber der alten Regelung ausgeweitet worden und erstreckt sich maximal auf drei Jahre, wenn (mindestens) sechs Monate durch den jeweils anderen Elternteil in Anspruch genommen werden. Ein zweimaliger Wechsel zwischen den Eltern ist möglich, allerdings können nicht beide Eltern gleichzeitig KBG beziehen.

Aufgrund der nach wie vor geringen Höhe der Transferleistung überwiegt der Anreiz zur traditionellen Rollenteilung zwischen den Eltern (Riesenfelder et al. 2007). So betrug der Anteil der Väter an allen KBG beziehenden Eltern 2008 nur 4 \% (BMG 2009). Die geringe Väterbeteiligung ergibt sich aber auch daraus, dass der „Verzicht" auf weitere sechs Monate KBG von den Eltern nicht als solcher wahrgenommen wird, da das KBG den Bezugszeitraum verlängerte: Konnte vorher ein Elternteil bis zu eineinhalb Jahre Erziehungsgeld beziehen, so sind jetzt maximal zweieinhalb Jahre möglich. Die Gewährung von sechs (zusätzlichen) Partnermonaten stellt kaum einen Anreiz zur Väterbeteiligung dar (Fritsch 2004).

Der KBG beziehende Elternteil darf jährlich bis zu $16.200 €$ brutto hinzuverdienen, ohne dass es zu Leistungskürzungen kommt. Diese Regelung bietet die Möglichkeit der eigenständigen Existenzsicherung für den erziehenden Elternteil und fördert die kontinuierliche Beschäftigungsintegration von Eltern (namentlich: Müttern), was sich positiv auf deren Wiedereinstiegschancen auswirken könnte. Allerdings werden dadurch flexible und ungeschützte Beschäftigungsformen gefördert (Schmidjell 2003) und somit wird die geschlechtsspezifische Segregation des Arbeitsmarktes verstärkt.

2 Zu den Umverteilungswirkungen des Elterngelds siehe kritisch Bothfeld (2006); Farahat et al. (2006); Henninger et al. (2008). 
Insgesamt betrachtet scheint sich die verlängerte Bezugsdauer des KBG negativer auf die Frauenerwerbstätigkeit auszuwirken, als durch die positiven Effekte der Zuverdienstmöglichkeit wettgemacht werden kann. So hat sich zwar der Anteil von Wiedereinsteigerinnen innerhalb des ersten Jahres deutlich erhöht: Während unter der alten Regelung nur $5 \%$ der Frauen innerhalb der ersten zwölf Monate wieder in eine Standardbeschäftigung einstiegen, sind es unter der KBG-Regelung 9\%. Gleichzeitig aber verringerte sich der Anteil der Frauen, die im zweiten Bezugsjahr in den Arbeitsmarkt zurückkehren: Waren es unter der alten Regelung $42 \%$, so sind es unter der KBG-Regelung nur noch $19 \%$. Mütter steigen also insgesamt länger aus der Erwerbsarbeit aus als früher. Es wird jedoch angenommen, dass sie langfristig in ähnlich hohem Ausmaß wie früher auf den Arbeitsmarkt zurückkehren werden (Riesenfelder et al. 2007). Ob sie nach dem längeren Ausstieg den Wiedereinstieg auch ähnlich gut bewältigen werden, ist umstritten (Lutz 2004). Dies auch deshalb, weil die arbeitsrechtliche Absicherung von KBGBezieherinnen und -Beziehern durch die Elternzeit sich nur bis zum zweiten Lebensjahr des Kindes erstreckt. Danach besteht keine rechtlich gesicherte Rückkehrmöglichkeit auf den alten Arbeitsplatz. Gleichzeitig wird ein früher Wiedereinstieg durch die unzureichende Betreuungssituation bei den Unter-Dreijährigen erschwert: Deren Betreuungsquote hat sich von 4,6\% im Jahr 1995 auf lediglich 11,8 \% im Jahr 2007 erhöht (Statistik Austria 2008).

Seit dem 1.1.2008 kann das KBG in drei unterschiedlichen Varianten bezogen werden: Alternativ zur bisherigen Regelung kann eine verkürzte Bezugszeit zu einem höheren monatlichen Pauschalsatz gewählt werden. Eltern haben die Möglichkeit, maximal für 20 Monate (24 bei Partnerbeteiligung) $624 €$ oder $800 €$ für längstens 15 Monate (18 bei Partnerbeteiligung) zu beziehen. Diese Regelung fördert einen rascheren Wiedereinstieg und führt zum Teil auch ohne Zuverdienst zur finanziellen Unabhängigkeit von Erziehenden. Entscheidend für die positive Umsetzung dieser neuen Optionen ist jedoch eine ausreichende Betreuungsinfrastruktur. Solange die Betreuung für Unter-Dreijährige nicht weiter ausgebaut wird, können nur wenige die Anreize zur Erwerbstätigkeit nutzen.

\subsection{FRANKREICH}

Das französische Erziehungsgeld („complément de libre choix d'activité") bietet eine Leistung für alle Eltern, die während der ersten drei Lebensjahre ihres zweiten (dritten, vierten usw.) Kindes ihre Erwerbstätigkeit ganz oder teilweise unterbrechen. Die Höhe der Leistung beträgt $552 €$ monatlich und kann seit 2004 auch für erstgeborene Kinder beansprucht werden, allerdings für längstens sechs Monate. Voraussetzung ist eine Erwerbstätigkeit von zwei Jahren vor der Geburt. Beim zweiten Kind sind zwei Jahre Erwerbstätigkeit innerhalb der letzten vier Jahre und beim dritten Kind innerhalb der letzten fünf Jahre vor der Geburt erforderlich.

Die Voraussetzung der vorangegangenen Erwerbstätigkeit schließt all diejenigen aus, die lange nicht oder nur kurze Zeit gearbeitet haben. Dies betrifft vor allem Frauen aus sozial benachteiligten Milieus, die häufigere und längere Erwerbsunterbrechungen aufgrund der Geburt von Kindern aufweisen als besser qualifizierte Frauen. Somit werden soziale Ungleichheiten verstärkt: Diejenigen, die es finanziell gut gebrauchen könnten, kommen kaum in den Genuss des Erziehungsgeldes, während es den besser gestellten Frauen sogar ohne Einkommensprüfung zur Verfügung steht. Es gibt aber auch Frauen, die Anspruch auf das Erziehungsgeld hätten, ihn aber nicht nutzen: Zum einen jene am unteren Ende des beruflichen Spektrums, die sich eine Erziehungsauszeit aus finanziellen Gründen nicht leisten können, und zum anderen hoch qualifizierte Frauen, die aus Karrieregründen auf eine Erziehungszeit verzichten. Mitte der 1990er Jahre bezogen lediglich $21 \%$ der Mütter mit zwei Kindern und $40 \%$ der Mütter mit drei und mehr Kindern Erziehungsgeld (Fagnani 1996).

Die geringe Höhe der Leistung führt auch in Frankreich in Kombination mit den geschlechtsspezifischen Einkommensungleichheiten zu einer geschlechtsspezifischen Inanspruchnahme von Erziehungsgeld: Der Väteranteil beträgt gerade einmal $1 \%$ (Rüling/Kassner 2007). Es sind vor allem junge und/oder gering qualifizierte Frauen, die das Erziehungsgeld in Anspruch nehmen: Zum einen diejenigen, die die Voraussetzung der zweijährigen Erwerbstätigkeit vor der Geburt innerhalb der Rahmenfrist erbringen können, weil es sich um das erste oder zweite Kind handelt.
Zum anderen diejenigen, für die aufgrund ihres geringen Verdienstes die Transferleistung interessanter ist als die Aufrechterhaltung der Erwerbsarbeit (Reuter 2002). Gleichzeitig ist das Erziehungsgeld nicht existenzsichernd, sodass diese Mütter jedenfalls auf einen Ernährer angewiesen sind. Allerdings wurde zum 1.7.2006 die Möglichkeit geschaffen, ab dem dritten Kind ein höheres Erziehungsgeld von $790 €$ monatlich für einen verkürzten Zeitraum von maximal zwölf Monaten in Anspruch zu nehmen (,complément optionnel de libre choix d'activité"). Diese Regelung korrespondiert mit einem vergleichsweise gut ausgebauten Betreuungsangebot für die Unter-Dreijährigen. Etwa die Hälfte der 40 \%igen Betreuungsquote garantieren dabei Tagesmütter und Kinderfrauen, deren Beschäftigung besonders gefördert wird.

Seit 2004 kann, wenn beide Eltern erwerbstätig sind, das sogenannte „complément de libre choix du mode de garde“ in Anspruch genommen werden. Dieses unterstützt die Fremdbetreuung von Kindern unter sechs Jahren durch eine Tagesmutter oder eine Kinderfrau. Dabei werden den Eltern als Arbeitgeber im Fall der Tagesmutter die gesamten, im Fall der Kinderfrau die Hälfte der Sozialabgaben erlassen. Zusätzlich wird ein Teil der Lohnkosten für die Tagesmutter oder die Kinderfrau erstattet. Die Höhe der Erstattung variiert nach dem Haushaltseinkommen, der Zahl der Kinder und dem Alter der Kinder. Für Unter-Dreijährige liegt sie aktuell zwischen $167 €$ und $442 €$, für Drei- bis Sechsjährige zwischen $84 €$ und $221 €$ pro Monat. Diese Politik der „Diversifizierung der Betreuungsformen“, die unter dem Diktum der Wahlfreiheit steht und in ähnlicher Form bereits seit Mitte der 1980er Jahre besteht, hat dazu geführt, dass sich in den Krippen zunehmend weniger Kinder der Mittel- und Oberschicht befinden und es zu einer sozialen Segregation des Betreuungssystems gekommen ist. Die Neuregelung der unterstützten Fremdbetreuung wird diese Segregationstendenzen fortführen (Fraisse/Eme 2005). Niedrigeinkommensbezieherinnen greifen mangels nicht ausreichender Krippenplätze oftmals auf nicht angemeldete Kinderfrauen zurück und, wenn vorhanden, auf Familienmitglieder. Alternativ unterbrechen sie ihre Erwerbstätigkeit, um ihre Kinder mithilfe des Erziehungsgelds selbst zu betreuen (Fagnani 1998, S. 598). 


\begin{tabular}{|c|c|c|c|}
\hline & Deutschland & Österreich & Frankreich \\
\hline Leistungshöhe pro Monat & $300-1.800 €$ & $436 / 618-800 €$ & $552-790 €$ \\
\hline Väterbeteiligung & $10,5 \%$ & $4 \%$ & $1 \%$ \\
\hline Dauer & 1 Jahr +2 Monate & $1,25-2,5$ Jahre & 0,5-3 Jahre \\
\hline $\begin{array}{l}\text { Betreuungsquote der } \\
\text { Unter-Dreijährigen }\end{array}$ & $18 \%$ & $12 \%$ & $40 \%$ \\
\hline
\end{tabular}

\subsection{GESCHLECHTS- UND SCHICHTSPEZIFISCHE WIRKUNGEN IM VERGLEICH}

Entscheidend für die Qualität einer sozialpolitischen Leistung für Erziehungsarbeit ist aus feministischer Perspektive die Frage, ob die Existenzsicherung der erziehenden Person gewährleistet wird (Becker-Schmidt/ Knapp 2000, S. 24ff.). In allen drei Ländern werden derartige Leistungen angeboten, allerdings jeweils nur für bestimmte Gruppen von Eltern:

- In Deutschland ist die Höhe der Leistung vom vorangegangenen Einkommen abhängig. Eltern mit weniger als $830 €$ Monatseinkommen erhalten deshalb kein existenzsicherndes Elterngeld. ${ }^{3}$

- In Österreich erhalten nur diejenigen ein existenzsicherndes Kinderbetreuungsgeld, die entweder eine gekürzte Bezugsdauer wählen oder einen Zuschuss aufgrund ihres niedrigen Haushaltseinkommens beantragen können.

- In Frankreich bietet nur die verkürzte Bezugsdauer beim dritten Kind die Möglichkeit, ein existenzsicherndes Erziehungsgeld zu bekommen.

Da in Deutschland die Bezugsdauer des Elterngelds bereits vergleichsweise kurz ist, könnte der österreichische Zuschuss zum Kinderbetreuungsgeld eine Lösung für die Existenzsicherung von Eltern mit geringem vorangegangenen Einkommen sein (Tabelle 1). Die Garantie der Existenzsicherung verringert zudem soziale Ungleichheiten im Zugang zum Elterngeld: Auch Geringverdienerinnen und Geringverdiener könnten sich dann die Erziehungsauszeit leisten.

Vom Standpunkt der Geschlechtergerechtigkeit aus stellt sich des Weiteren die Frage, inwiefern die Regelungen eine partnerschaftliche Aufteilung der Erziehungsarbeit zwischen den Eltern unterstützen. Anreize zur Beteiligung von Vätern an der Erziehungsarbeit werden so- wohl in Deutschland als auch in Österreich gesetzt, allerdings scheint die deutsche Variante der Partnermonate bessere Ergebnisse zu zeitigen. Dies liegt an der im Durchschnitt großzügigeren Leistungshöhe in Deutschland sowie an der insgesamt vergleichsweise langen Bezugsdauer für einen Elternteil in Österreich.

Die maximale Bezugsdauer ist zudem ein wichtiger Indikator für die Chancen des Wiedereinstiegs von Erziehenden in den Arbeitsmarkt. Insbesondere vor dem Hintergrund einer angespannten Arbeitsmarktsituation gestaltet sich die Rückkehr in die Erwerbsarbeit nach langen Ausstiegen in der Regel problematisch. Insofern gibt es in allen drei Ländern Anreize für einen kurzen Erwerbsausstieg von Eltern: Ein Jahr in Deutschland, 15 oder 20 Monate in Österreich, sechs Monate beim ersten Kind und ein Jahr beim dritten Kind in Frankreich. Gleichzeitig jedoch besteht in allen drei Ländern die Möglichkeit eines zum Teil deutlich längeren Erwerbsausstiegs für Eltern: Bis zu drei Jahre in Deutschland und Frankreich sowie bis zu zweieinhalb Jahre in Österreich. Hieran zeigt sich m. E., dass sich in allen drei Ländern zur Zeit ein Umbruch im Geschlechterverhältnis abzeichnet: Zum einen werden an der traditionellen weiblichen Geschlechterrolle orientierte lange Auszeiten ermöglicht, die jedoch wegen der damit verbundenen Leistungshöhe nur für diejenigen Eltern infrage kommen, die sich derartig lange Ausstiege leisten können. Zum anderen werden Anreize für kurze Auszeiten und eine modernisierte weibliche (oder auch männliche) Geschlechterrolle gesetzt, die aber wiederum nur dann eine realistische Option für Eltern darstellen, wenn eine ausreichende Betreuungssituation für Kinder unter drei Jahren gegeben ist.

Frankreich weist die höchste Betreuungsquote der Unter-Dreijährigen auf und kann somit als einziges der drei Länder tatsächlich ein gewisses Maß an Wahlfreiheit zwischen unterschiedlichen Geschlechterrollen ermöglichen. Wenn der
Ausbau der Kinderbetreuung für UnterDreijährige wie geplant voranschreitet, wird dies auch für Deutschland der Fall sein. Allerdings ist zu bedenken, dass in Frankreich aufgrund der steuer- und sozialpolitischen Förderung von Tagesmüttern und Kindermädchen eine schichtspezifische Segregation in der Kinderbetreuung entstanden ist. Deutschland sollte deshalb von einer Orientierung an dieser speziellen Förderung, wie sie im Fall der steuer- und abgabenbegünstigten „haushaltsnahen Dienstleistungen "bereits angelegt ist, absehen.

\section{3 \\ Altenpflegepolitiken in den Sozialversicherungs- ländern}

Die Pflege von älteren Menschen findet in allen drei Vergleichsländern nur in geringem Maße außerhalb der Familie statt. Etwa zwei Drittel aller pflegebedürftigen älteren Menschen sind - zu einem großen Teil ausschließlich - auf die Unterstützung und Betreuung durch Angehörige angewiesen. Diese werden in den einzelnen Ländern jedoch auf unterschiedliche Art und Weise sozialpolitisch unterstützt.

\subsection{DEUTSCHLAND}

Mit dem Pflegegeld stellt die Pflegeversicherung im Fall der häuslichen Pflege eine Geldleistung zur Verfügung, die je nach Grad der Pflegebedürftigkeit zwischen $215 €$ und $675 €$ monatlich beträgt. Das Pflegegeld stellt damit nur in der Pflegestufe III einen existenzsichernden Betrag dar. Hinzu kommt, dass die Geldleistung zwar für die familiale Pflegeperson gedacht ist, jedoch keine direkte Zahlung an die Pflegeperson stattfindet, d.h. die Pflegeperson ist darauf angewiesen, dass die pflegebedürftige Person das Pflegegeld entsprechend weiterreicht. Angesichts des steigenden Pflegeaufwands von Pflegestufe I zu III und dem nicht proportional steigenden Pflegegeld

3 Das soziokulturelle Existenzminimum, welches sich aus dem Regelsatz für die Grundsicherung für Arbeitslose plus den durchschnittlichen Kosten für Wohnen und Heizung zusammensetzt, liegt bei $625 €$ pro Monat. Trotz des GeringverdienendenZuschlags des Elterngelds würde ab einem Einkommen von unter $830 €$ das Elterngeld unterhalb des soziokulturellen Existenzminimums liegen. 
gestaltet sich zudem die monetäre Bewertung von Pflegearbeit umso dürftiger, je belastender die Pflege wird. Allerdings sind pflegende Angehörige, wenn sie mindestens 14 Stunden pro Woche Pflegearbeit leisten, in der Unfall- und der Rentenversicherung abgesichert. Bezogen auf die (potenziell) erwerbstätige Bevölkerung stellt das Pflegegeld einen Anreiz zur Fortschreibung der geschlechtsspezifischen Arbeitsteilung in der familialen Altenpflege dar. So sind etwa drei Viertel der Hauptpflegepersonen in der häuslichen Pflege (Ehe-)Partnerinnen, Töchter oder Schwiegertöchter (BMFSFJ 2005).

Seit dem 1.7.2008 können Beschäftigte eine unbezahlte kurzzeitige Freistellung von zehn Arbeitstagen in Anspruch nehmen, um bei Eintritt einer akuten Pflege-Notsituation Pflege für Angehörige zu organisieren. Zudem können Arbeitnehmer und Arbeitnehmerinnen in Betrieben mit mehr als 15 Beschäftigten eine unbezahlte Pflegezeit von sechs Monaten in Anspruch nehmen. Ihr Sozialversicherungsschutz bleibt während dieser Zeit erhalten, und es besteht, ähnlich wie bei der Elternzeit, ein Kündigungsschutz. Damit wird zwar eine begrenzte Möglichkeit zur Vereinbarkeit von Pflege und Erwerbstätigkeit geschaffen, diese kann jedoch nur bei einer entsprechenden finanziellen Absicherung der Pflegeperson über das Pflegegeld oder einen Partner bzw. eine Partnerin wahrgenommen werden. Die relativ kurze Dauer der Pflegezeit könnte in Kombination mit der Möglichkeit, dass verschiedene Angehörige diese nacheinander in Anspruch nehmen, zu einer Aufteilung der Pflege auf mehrere Angehörige führen und einen Schritt zu mehr Geschlechtergerechtigkeit darstellen. Im schlechtesten Fall fördert die Pflegezeit durch das zeitlich begrenzte Rückkehrrecht den dauerhaften Erwerbsausstieg von pflegenden Angehörigen.

\section{2 ÖSTERREICH}

In Österreich besteht unabhängig von vorangegangener Beitragsleistung und von Einkommens- und Vermögensverhältnissen Anspruch auf ein steuerfinanziertes Pflegegeld, wenn ein ständiger Pflegebedarf vorliegt, der voraussichtlich mindestens sechs Monate dauern wird und monatlich mehr als 50 Stunden beträgt. Je nach Ausmaß des Pflegebedarfs staffelt sich die Höhe des Pflegegelds in sieben Stufen zwischen $154 €$ und $1.656 €$ monatlich. Das
Pflegegeld wird an die pflegebedürftige Person ausbezahlt, die über die Verwendung der Geldleistung autonom entscheiden kann. ${ }^{4}$ Pflegende Angehörige erhalten nur dann eine Vergütung für ihre Pflegearbeit, wenn die pflegebedürftige Person das Pflegegeld entsprechend weiterreicht. Zwar haben $86 \%$ der familialen Pflegepersonen zumindest einen indirekten Zugang zum Pflegegeld (Badelt/Österle 1997, S. 193), ${ }^{5}$ dieses wird jedoch zumeist dazu verwendet, soziale Dienste zu bezahlen, Anpassungen in der Wohnung vorzunehmen oder spezielle Hilfsmittel zu kaufen. Für die Pflegepersonen selbst bleibt in der Regel nur eine symbolische Zahlung übrig. Insofern bietet das Pflegegeld keinen Anreiz für eine Entgeschlechtlichung der Angehörigenpflege. Es verstärkt im Gegenteil den Druck auf Frauen, ihrer erwarteten Rolle als unbezahlte Pflegeperson in der Familie nachzukommen (Hammer/Österle 2003, S. 46f.), zumal das geringe Leistungsniveau des Pflegegelds die Finanzierung einer bedarfsgerechten, professionellen Pflege erheblich erschwert.

Seit 2002 ermöglicht die sogenannte Familienhospizkarenz die Unterbrechung der Erwerbstätigkeit zugunsten familialer Pflegearbeit. Zum Zweck der Sterbebegleitung von Angehörigen können Arbeitnehmer und Arbeitnehmerinnen eine Herabsetzung der Arbeitszeit, eine Änderung der Lage der Arbeitszeit oder eine unbezahlte Freistellung von der Arbeitsleistung für längstens sechs Monate verlangen. Nach Ende der Familienhospizkarenz besteht ein viermonatiger Kündigungsschutz. Arbeitnehmer und Arbeitnehmerinnen, die aufgrund der Inanspruchnahme von Familienhospizkarenz kein oder nur ein geringfügiges Entgelt erhalten, werden kostenfrei krankenversichert (Sachleistungsversicherung) und erhalten Beitragszeiten in der Rentenversicherung gutgeschrieben. In besonderen Härtefällen kann im Fall der unbezahlten Freistellung eine Geldzuwendung gewährt werden, um Bezieher und Bezieherinnen niedriger Einkommen nicht zu benachteiligen: Wenn das gewichtete Haushaltseinkommen pro Person unter $700 €$ pro Monat sinkt, wird es auf dieses Minimum angehoben.

\subsection{FRANKREICH}

In Frankreich existiert ein steuerfinanziertes Pflegegeld („allocation personnalisée d'autonomie“), dessen Höhe vom Grad der
Pflegebedürftigkeit und vom Einkommen der pflegebedürftigen Person abhängt. Der Maximalbetrag liegt in der niedrigsten Pflegestufe bei $520 €$ und in der höchsten bei $1.213 €$. Den Maximalbetrag bekommen Pflegebedürftige, deren Einkommen unter $683 €$ pro Monat beträgt. Bei einem monatlichen Einkommen zwischen $683 €$ und $2.722 €$ haben die Pflegebedürftigen einen Selbstbehalt zu tragen, der linear von $0 \%$ auf $90 \%$ ansteigt. Liegt das Einkommen über $2.722 €$ pro Monat, beträgt der Selbstbehalt in jedem Fall 90 \%. Für jede pflegebedürftige Person wird ein persönlicher Pflegeplan erstellt, in dem die Verwendung des Pflegegelds genau festgelegt wird. Die Bezahlung von Pflegegeld an pflegende Angehörige ist dabei möglich, allerdings erhalten nur $8 \%$ der pflegenden Angehörigen Pflegegeld. Nur in den beiden höchsten Pflegestufen kann das Pflegegeld einen Betrag erreichen, der als existenzsichernd für pflegende Angehörige bezeichnet werden kann. Es bleibt jedoch zu berücksichtigen, dass nur wenige Pflegebedürftige in den beiden höchsten Stufen sind und den dafür angesetzten Maximalbetrag erhalten (Petite/Weber 2006).

Seit 2007 besteht für erwerbstätige pflegende Angehörige, die mindestens zwei Jahre bei demselben Arbeitgeber beschäftigt waren, die Möglichkeit, eine unbezahlte Freistellung von drei bis zu maximal zwölf Monaten im Sinne einer Sterbebegleitung in Anspruch zu nehmen („,congé de soutien familial“). Während dieser Zeit kann eine beitragsfreie Rentenversicherung gewährt werden, wenn das Haushaltseinkommen niedrig ist. Nach Ende der Beurlaubung hat der Arbeitnehmer bzw. die Arbeitnehmerin das Recht, auf den alten oder einen gleichwertigen Arbeitsplatz zurückzukehren.

\subsection{GESCHLECHTS- UND SCHICHTSPEZIFISCHE WIRKUNGEN IM VERGLEICH}

Die familiale Altenpflegearbeit wird zu etwa einem Drittel von Personen geleistet,

\footnotetext{
4 Im Fall einer stationären Unterbringung erhält die pflegebedürftige Person nur ein Taschengeld in Höhe von $10 \%$ der Pflegestufe 3 (ca. $44 €$ pro Monat), der restliche Betrag wird direkt an das entsprechende Alten- oder Pflegeheim ausbezahlt.

$539 \%$ erhalten eine regelmäßige, $20 \%$ eine fallweise finanzielle Entschädigung, $27 \%$ haben eine gemeinsame Kasse mit der pflegebedürftigen Person.
} 
Tabelle 2: Altenpflegepolitiken im Vergleich, ausgewählte Indikatoren (2009)

\begin{tabular}{lccc}
\hline & Deutschland & Österreich & Frankreich \\
\hline Leistungshöhe pro Monat & $215-675 €$ & $154-1.656 €$ & $520-1.213 €$ \\
Pflegezeit & 10 Tage +6 Monate & 6 Monate & 12 Monate \\
\hline & & WSI MITTEILUNGEN \\
Quelle: Zusammenstellung der Autorin. & & & WS
\end{tabular}

die selbst bereits im Rentenalter sind und über eine existenzsichernde monatliche Rentenzahlung verfügen. Der größte Teil der Angehörigenpflege wird jedoch von Frauen (namentlich: Töchtern und Schwiegertöchtern) im erwerbsfähigen Alter erbracht, die zunehmend erwerbstätig sind. Für sie bedeutet die Übernahme von Pflegeverantwortung in der Regel, dass sie ihre Arbeitszeit reduzieren oder ihre Erwerbstätigkeit (vorübergehend) aufgeben und dadurch einen massiven Einkommensverlust erfahren. Die Leistungen für pflegende Angehörige können diesen Effekt nur partiell auffangen, da ihre Höhe nur in den oberen Pflegestufen als existenzsichernd bezeichnet werden kann. Die Alternative der Teilzeit-Erwerbstätigkeit neben der Angehörigenpflege ist nur bei der Pflege von leicht pflegebedürftigen Angehörigen eine Option zur eigenständigen Existenzsicherung der Pflegeperson. Je stärker die Pflegebedürftigkeit der gepflegten Person ansteigt, desto weniger Erwerbsarbeit kann von der Pflegeperson geleistet werden. Es entsteht eine „Existenzsicherungsschere“ im mittleren Bereich der Pflegebedürftigkeit, wenn das Pflegegeld nicht existenzsichernd ist und die zusätzliche Teilzeit-Erwerbstätigkeit dieses Defizit nicht ausgleichen kann.

Bei einer längeren Unterbrechung der Erwerbstätigkeit aufgrund von Angehörigenpflege wird die spätere Rückkehr auf den Arbeitsmarkt schwierig; das Reintegrationsproblem verschärft sich mit steigendem Alter. Die mittlerweile in allen drei Ländern vorhandenen Möglichkeiten zur Pflegefreistellung mit anschließendem Kündigungsschutz bieten eine wesentliche Verbesserung für pflegende Angehörige. Allerdings handelt es sich dabei in Deutschland wie in Österreich um eine relativ kurze Freistellung von sechs Monaten. Eine Ausweitung auf zwölf Monate wie in Frank- reich wäre nicht nur im Interesse der pflegenden Angehörigen wünschenswert, sondern würde auch eine Angleichung an die bislang besseren Bedingungen der familialen Kinderbetreuungsarbeit bedeuten.

Aus ungleichheitssoziologischer Perspektive müsste die finanzielle Absicherung während der Pflegefreistellung garantiert werden. In Deutschland ist diese Absicherung nur bedingt gegeben, wenn (a) das Pflegegeld an die pflegende Person weitergegeben wird und (b) das Pflegegeld über dem Existenzminimum liegt, die zu pflegende Person also in der Pflegestufe III eingestuft ist und keine Sachleistungen für die häusliche Pflege aus der Pflegeversicherung bezogen werden. Aus der Perspektive der pflegenden Angehörigen müsste demnach, ähnlich wie dies in Frankreich bei der Festlegung des persönlichen Pflegeplans der Fall ist, zunächst sichergestellt werden, dass sie das ihnen für die geleistete Pflege zustehende Pflegegeld auch tatsächlich erhalten. Zudem müsste ein Modus gefunden werden, der denjenigen pflegenden Angehörigen, die nicht auf ein existenzsicherndes Pflegegeld zurückgreifen können, ein solches garantiert. Dabei könnte man sich an der österreichischen „Härtefallregelung“ im Rahmen der Familienhospizkarenz orientieren (Abschnitt 3.2).

\section{1 \\ Fazit: Von den Nachbarn lernen?}

Der statusreproduzierende Charakter des deutschen Wohlfahrtsstaats spiegelt sich auch in der Kinderbetreuungs- und Altenpflegepolitik und führt dort zu schichtspezifischen Diskriminierungsstrukturen bezüglich der Möglichkeit, familiale Kinderbetreuungs- und Altenpflegearbeit als Al- ternative zur Erwerbstätigkeit zu leben. Um die Entscheidung für Familienarbeit nicht von der individuellen Einkommens- und Beziehungssituation abhängig zu machen, könnte

(a) das Elterngeld für Niedrigeinkommensbezieher und -bezieherinnen auf das soziokulturelle Existenzminimum angehoben werden und

(b) das Pflegegeld in Höhe des soziokulturellen Existenzminimums direkt an die pflegenden Angehörigen ausbezahlt werden. Dabei wäre die Möglichkeit der Teilzeit-Erwerbstätigkeit bei leichterer Pflegebedürftigkeit ebenso zu berücksichtigen wie die Notwendigkeit pflegende Angehörige durch Sachleistungen für die häusliche Pflege zu unterstützen.

Die individuelle Existenzsicherung von Familienarbeitenden würde nicht nur das „Recht auf Familienarbeit" für alle sozialen Schichten ermöglichen, sondern auch die finanzielle Unabhängigkeit der Familienarbeitenden von der Familie. In Kombination mit der arbeitsrechtlich abgesicherten Rückkehr in den Arbeitsmarkt werden fließende Übergänge zwischen Familienund Erwerbsarbeit ermöglicht, wobei hier die Rechte der pflegenden Angehörigen im Vergleich zu den Erziehenden noch deutlich schlechter ausgestaltet sind und im Sinne der Gleichbehandlung von Familienarbeitenden angeglichen werden müssten.

Die Option Betreuungs- und Pflegearbeit aus der Familie auszulagern ist im deutschen Wohlfahrtsstaat traditionell nur sehr begrenzt gegeben. Während sich für die Kinderbetreuung eine neue Entwicklung bereits abzeichnet, ist in der Altenpflege kein der demografischen Entwicklung entsprechender Ausbau festzustellen: Pflegende Angehörige (und im Übrigen auch Pflegebedürftige) haben kaum Wahlmöglichkeiten. Das „Recht, nicht zu pflegen“ (Lewis 1987), kann in keinem der Bismarckschen Wohlfahrtsstaaten eingelöst werden. Hierfür wären mögliche Vorbilder wohl weiter im Norden Europas zu finden. 


\section{LITERATUR}

Badelt, Ch./Österle, A. (1997): Pflegegeld, informelle Pflegearbeit und öffentliche Pflegeausgaben: Erfahrungen aus Österreich, in: Sozialer Fortschritt 8, S. 189-194

Becker-Schmidt, R./Knapp, G.-A. (2000): Feministische Theorien zur Einführung, Hamburg

Bothfeld, S. (2006): Das Elterngeld - Einige Anmerkungen zum Unbehagen mit der Neuregelung, in: femina politica 2, S. 102-107

Bruning, G./Platenga, I. (1999): Parental Leave and Equal Oppertunities: Experiences in Eight European Countries, in: Journal of European Social Policy 3, S. 195-209

Bundesministerium für Familie, Senioren, Frauen und Jugend (BMFSFJ) (2005): Möglichkeiten und Grenzen selbstständiger Lebensführung in Privathaushalten, Ergebnisse der Studie MuG III, Bonn Bundesministerium für Familie, Senioren, Frauen und Jugend (BMFSFJ) (2008): Dossier Elterngeld als Teil nachhaltiger Familienpolitik, Berlin Bundesministerium für Gesundheit (BMG) (2009): Kinderbetreuungsgeld-Statistik, www.bmgfj.gv.at, letzter Zugriff: 6.2.2009

Ehnis, P. (2008): Hegemoniale Mütterlichkeit. Vom selbstverständlichen Einverständnis in die geschlechtstypische Arbeitsteilung nach der Geburt eines Kindes, in: Marburger Gender-Kolleg (Hrsg.): Geschlecht Macht Arbeit. Interdisziplinäre Perspektiven und politische Intervention, Münster, S. 56-69

Fagnani, J. (1996): L'allocation parentale d'éducation: contraintes et limites du choix d'une prestation, in: Lien social et politiques - RIAC 3 , S. $111-121$

Fagnani, J. (1998): Lacunes, contradictions et incohérences des mesures de conciliation travail/famille, in: Droit social 6, S. 596-602

Farahat, A./Janczyk, S./Mängel, A./Schönig, B. (2006): Exklusive Emanzipation. Zur Frauen- und Familienpolitik der großen Koalition, in: Blätter für deutsche und internationale Politik 8, S. 985-994

Fraisse, L./Eme B. (2005): La gouvernance locale de la diversification des modes d'accueil : un enjeu de cohésion sociale. Recherches et Prévisions, CNAF, n80, june 2005

Fritsch, C. (2004): Zu Risiken oder Nebenwirkungen lesen Sie den Gesetzestext oder fragen Sie Ihren Steuerberater. Intention und Wirkung des Kinderbetreuungsgeldgesetzes in einem breiten Kontext, Dissertationsschrift, Wien

Hammer, E./Österle, A. (2003): Welfare State Policy and Informal LongTerm Care Giving in Austria: Old Gender Divisions and New Stratification Processes Among Women, in: Journal of Social Policy 1, S. 37-53
Henninger, A./Wimbauer, Ch./Dombrowski, R. (2008): Geschlechtergleichheit oder "exklusive Emanzipation"? Ungleichheitssoziologische Implikationen der aktuellen familienpolitischen Reformen, in: Berliner Journal für Soziologie 1, S. 99-128

Jenson, J./Jacobzone, S. (2000): Care Allowances for the Frail Elderly and Their Impact on Women Care-Givers. Labour Market and Social Policy Occasional Papers No. 41, Paris

Knijn, T./Kremer, M. (1997): Gender and the Caring Dimension of Welfare States: Toward Inclusive Citizenship, in: Social Politics 3, S. 328-361 Leitner, S. (2008): Varianten von Familialismus. Eine historisch vergleichende Analyse der Kinderbetreuungs- und Altenpflegepolitiken in kontinentaleuropäischen Wohlfahrtsstaaten, Habilitationsschrift, Göttingen

Lewis, I. (1987): Gender and Welfare Regimes: Further Thoughts, in : Social Politics 2, S. 160-177

Lutz, H. (2004): Wiedereinstieg und Beschäftigung von Frauen mit Kleinkindern. Ein Vergleich der bisherigen Karenzregelung mit der Übergangsregelung zum Kinderbetreuungsgeld, Wien

Petite, S./Weber, A. (2006): Les effets de l'Allocation personnalisée d'autonomie sur l'aide dispensée aux personnes âgées, Études et Résultats 459, Paris

Reuter, S. (2002): Frankreichs Wohlfahrtsregime im Wandel? Erwerbsintegration von Französinnen und familienpolitische Reformen der 90er Jahre, ZeS-Arbeitspapier 13, Bremen

Riesenfelder, A./Sorger, C./Wetzel, P./Willsberger, B. (2007): Das Kinderbetreuungsgeld in Österreich. Auswirkungen auf das Erwerbsverhalten und die Beschäftigungsfähigkeit, Wien

Rüling, A. (2008): Ein Jahr Elterngeld - Geschlechterrevolution oder Leistung für Besserverdienende?, in: femina politica 1, S. 115-118

Rüling, A./Kassner, K. (2007): Familienpolitik aus der Gleichstellungsperspektive. Ein europäischer Vergleich, Berlin

Schmidjell, C. (2003): Kinderbetreuungsgeld: Vor- oder Nachteil für Frauen? Vor- oder Nachteil für Arbeitnehmerlnnen?, in: Aichhorn, U. (Hrsg.): Unterhalt - Obsorge - Kinderbetreuungsgeld: aus frauen(rechtlicher) Perspektive, Wien, S. 67-77

Statistik Austria (2008): Kindertagesheimstatistik 2007/08, Wien Statistisches Bundesamt (2008): 18 \% der Kinder unter drei Jahren in Tagesbetreuung. Pressemitteilung Nr. 495 vom 18.12., www.destatis.de, letzter Zugriff: 6.2.2009

Ungerson, C. (1995): Gender, Cash and Informal Care: European Perspectives and Dilemmas, in: Journal of Social Policy 1, S. 31-52 